\title{
Inflation Hedging and Industry Stock Returns
}

\author{
Tai D. Yi
}

The BRC Academy Journal of Business 7, no. 1 (2017): 1-22.

http://dx.doi.org/10.15239/j.brcacadjb.2017.07.01.ja01

\section{Web APPENDIX}

http://dx.doi.org/10.15239/j.brcacadjb.2017.07.01.wa01 
Table 1

Augmented Dickey-Fuller Unit Root Tests for the VAR Model

The null hypothesis $\left(\mathrm{H}_{0}\right)$ is that the time-series has a unit root and thus is nonstationary while the alternative hypothesis $\left(\mathrm{H}_{\mathrm{a}}\right)$ is that it is stationary. The sample period is 1959Q1 to $2014 \mathrm{Q} 4$.

Panel A: Tests for the undifferenced time series

Variables

$\operatorname{Tau}(\tau) \quad$ p-value

Growth rate of GDP - seasonally adjusted

$-5.79921$

0.00007

Growth rate of $\mathrm{M} 2$ - seasonally adjusted

$-4.75978$

0.00015

Growth rate of employ in mfg - seasonally adj.

$-5.53162$

0.00007

CPI inflation rate

$-2.65419$

0.08422

Growth rate of wages

$-1.88169$

0.34027

Growth rate of import prices

$-5.35635$

0.00007

Panel B: Tests for the first differences of CPI inflation rate and growth rate of wages

\begin{tabular}{lcc}
\hline Variables & Tau $(\tau)$ & p-value \\
\hline First difference of CPI inflation rate & -10.8059 & 0.00007 \\
First difference of growth rate of wages & -12.3185 & 0.00007 \\
\hline
\end{tabular}


Table 2

Parameter Estimates for the $\Delta \pi_{\mathrm{t}}$ Equation in the VAR Model

The table reports the parameter estimates for the $\Delta \pi_{\mathrm{t}}$ equation in the VAR model, where y is the growth rate of GDP, $\mathrm{m}$ is the growth rate of M2, $\mathrm{u}$ is the growth rate of employment, $\Delta \pi_{\mathrm{t}-1}$ is the first difference of the inflation rate, $\Delta \mathrm{w}_{\mathrm{t}-1}$ is the first difference of the growth rate of wages, and $\mathrm{pm}$ is the growth rate of import prices. The sample period is 1959Q2 to 2014Q4. *,**, and *** denote significance at the $10 \%$, $5 \%$, and $1 \%$ level, respectively.

\begin{tabular}{|c|c|c|c|c|c|}
\hline \multicolumn{2}{|l|}{ Panel A } & \multicolumn{2}{|c|}{ Parameter Estimate } & \multicolumn{2}{|c|}{ Standard Deviation } \\
\hline Intercept & & \multicolumn{2}{|c|}{-0.00135} & \multicolumn{2}{|c|}{0.0010} \\
\hline $\mathrm{y}_{\mathrm{t}-1}$ & & \multicolumn{2}{|c|}{-0.02099} & \multicolumn{2}{|c|}{0.0623} \\
\hline $\mathrm{y}_{\mathrm{t}-2}$ & & \multicolumn{2}{|c|}{0.01342} & \multicolumn{2}{|c|}{0.0617} \\
\hline $\mathrm{y}_{\mathrm{t}-3}$ & & \multicolumn{2}{|c|}{0.09327} & \multicolumn{2}{|c|}{0.0608} \\
\hline $\mathrm{y}_{\mathrm{t}-4}$ & & \multicolumn{2}{|c|}{0.07268} & \multicolumn{2}{|c|}{0.0550} \\
\hline $\mathrm{m}_{\mathrm{t}-1}$ & & \multicolumn{2}{|c|}{-0.04799} & \multicolumn{2}{|c|}{0.0501} \\
\hline $\mathrm{m}_{\mathrm{t}-2}$ & & \multicolumn{2}{|c|}{0.04147} & \multicolumn{2}{|c|}{0.0532} \\
\hline$m_{t-3}$ & & \multicolumn{2}{|c|}{-0.01725} & \multicolumn{2}{|c|}{0.0533} \\
\hline $\mathrm{m}_{\mathrm{t}-4}$ & & \multicolumn{2}{|c|}{-0.00475} & \multicolumn{2}{|c|}{$0.0494 *$} \\
\hline $\mathrm{u}_{\mathrm{t}-1}$ & & \multicolumn{2}{|c|}{0.25585} & \multicolumn{2}{|c|}{$0.1542 *$} \\
\hline $\mathrm{u}_{\mathrm{t}-2}$ & & \multicolumn{2}{|c|}{-0.17754} & \multicolumn{2}{|c|}{0.1823} \\
\hline $\mathrm{u}_{\mathrm{t}-3}$ & & \multicolumn{2}{|c|}{-0.10303} & \multicolumn{2}{|c|}{0.1843} \\
\hline $\mathrm{u}_{\mathrm{t}-4}$ & & 0.15 & & & \\
\hline$\Delta \pi_{\mathrm{t}-1}$ & & -0.74 & & & \\
\hline$\Delta \pi_{\mathrm{t}-2}$ & & -0.93 & & & \\
\hline$\Delta \pi_{\mathrm{t}-3}$ & & -0.47 & & & \\
\hline$\Delta \pi_{\mathrm{t}-4}$ & & -0.22 & & & \\
\hline$\Delta \mathrm{w}_{\mathrm{t}-1}$ & & 0.06 & & & \\
\hline$\Delta \mathrm{w}_{\mathrm{t}-2}$ & & 0.20 & & & \\
\hline$\Delta \mathrm{w}_{\mathrm{t}-3}$ & & 0.20 & & & \\
\hline$\Delta \mathrm{w}_{\mathrm{t}-4}$ & & 0.11 & & & \\
\hline $\mathrm{pm}_{\mathrm{t}-1}$ & & 0.08 & & & \\
\hline $\mathrm{pm}_{\mathrm{t}-2}$ & & -0.02 & & & \\
\hline $\mathrm{pm}_{\mathrm{t}-3}$ & & 0.00 & & & \\
\hline $\mathrm{pm}_{\mathrm{t}-4}$ & & -0.06 & & & \\
\hline Panel B & Mean & Median & Std Dev & Min & Max \\
\hline 1961Q2 - 2014Q4 & & & & & \\
\hline$\pi_{\mathrm{t}}$ & 0.0097 & 0.0084 & 0.0081 & -0.0286 & 0.0380 \\
\hline $\mathrm{E}\left(\pi_{\mathrm{t}}\right)$ & 0.0074 & 0.0058 & 0.0072 & -0.0095 & 0.0354 \\
\hline 1961Q2 - 1982Q4 & & & & & \\
\hline$\pi_{\mathrm{t}}$ & 0.0138 & 0.0118 & 0.0091 & 0.0000 & 0.0380 \\
\hline $\mathrm{E}\left(\pi_{\mathrm{t}}\right)$ & 0.0109 & 0.0097 & 0.0093 & -0.0041 & 0.0354 \\
\hline 1983Q1 - 2001Q4 & & & & & \\
\hline$\pi_{\mathrm{t}}$ & 0.0078 & 0.0078 & 0.0038 & -0.0028 & 0.0172 \\
\hline $\mathrm{E}\left(\pi_{\mathrm{t}}\right)$ & 0.0057 & 0.0051 & 0.0033 & -0.0022 & 0.0166 \\
\hline 2002Q1 - 2014Q4 & & & & & \\
\hline$\pi_{\mathrm{t}}$ & 0.0055 & 0.0054 & 0.0077 & -0.0286 & 0.0217 \\
\hline $\mathrm{E}\left(\pi_{\mathrm{t}}\right)$ & 0.0042 & 0.0040 & 0.0048 & -0.0095 & 0.0167 \\
\hline
\end{tabular}


Table 3

SIC Codes

NoDur Consumer NonDurables -- Food, Tobacco, Textiles, Apparel, Leather, Toys

0100-0999, 2000-2399, 2700-2749, 2770-2799, 3100-3199, 3940-3989

Durbl Consumer Durables -- Cars, TV's, Furniture, Household Appliances

2500-2519, 2590-2599, 3630-3659, 3710-3711, 3714-3714, 3716-3716, 3750-3751, 3792-3792, 3900-3939, 3990-3999

Manuf Manufacturing -- Machinery, Trucks, Planes, Off Furn, Paper, Com Printing 2520-2589, 2600-2699, 2750-2769, 3000-3099, 3200-3569, 3580-3629, 3700-3709, $3712-3713$, 3715-3715, 3717-3749, 3752-3791, 3793-3799, 3830-3839, 3860-3899

Enrgy Oil, Gas, and Coal Extraction and Products

1200-1399, 2900-2999

Chems Chemicals and Allied Products

2800-2829, 2840-2899

BusEq Business Equipment -- Computers, Software, and Electronic Equipment 3570-3579, 3660-3692, 3694-3699, 3810-3829, 7370-7379

Telcm Telephone and Television Transmission 4800-4899

Utils Utilities

4900-4949

Shops Wholesale, Retail, Services, Hotels, and Entertainment

5000-5999, 7000-7369, 7380-7699, 7800-7999, 8100-8999

Hlth Healthcare, Medical Equipment, and Drugs

2830-2839, 3693-3693, 3840-3859, 8000-8099

Money Finance

6000-6999

Mines Non-Metallic and Industrial Metal Mining 1000-1119, 1400-1499

Cnstr Construction and Some Construction Materials

1500-1511, 1520-1549, 1600-1799, 2400-2439, 2450-2459, 2490-2499

Trans Transportation

4000-4789 
Table 4

Summary Statistics

Panel A: Industry quarterly return, market to book ratio, return on assets, and leverage ratio, 1961Q2 - 2014Q4

\begin{tabular}{lccccc}
\multicolumn{7}{c}{ Quarterly Return } \\
\hline Industry & Mean & Median & Std Dev & Min & Max \\
\hline NoDur & 0.037 & 0.035 & 0.118 & -0.320 & 0.503 \\
Utils & 0.031 & 0.033 & 0.071 & -0.207 & 0.253 \\
Hlth & 0.046 & 0.041 & 0.146 & -0.346 & 0.565 \\
Durbl & 0.039 & 0.032 & 0.148 & -0.408 & 0.671 \\
Manuf & 0.037 & 0.040 & 0.127 & -0.348 & 0.428 \\
Chems & 0.038 & 0.041 & 0.112 & -0.341 & 0.375 \\
Shops & 0.038 & 0.035 & 0.136 & -0.328 & 0.601 \\
Mines & 0.037 & 0.031 & 0.158 & -0.376 & 0.772 \\
Cnstr & 0.038 & 0.026 & 0.165 & -0.377 & 0.884 \\
\hline
\end{tabular}

Market to Book Ratio

\begin{tabular}{lccccc}
\hline Industry & Mean & Median & Std Dev & Min & Max \\
\hline NoDur & 1.596 & 1.717 & 0.410 & 0.544 & 2.241 \\
Utils & 1.236 & 1.298 & 0.282 & 0.634 & 1.723 \\
Hlth & 2.505 & 2.587 & 0.477 & 1.187 & 4.588 \\
Durbl & 1.581 & 1.685 & 0.407 & 0.409 & 2.280 \\
Manuf & 1.521 & 1.575 & 0.368 & 0.605 & 2.211 \\
Chems & 1.845 & 1.942 & 0.435 & 0.708 & 2.539 \\
Shops & 1.720 & 1.848 & 0.433 & 0.589 & 2.320 \\
Mines & 1.759 & 1.739 & 0.456 & 0.793 & 2.923 \\
Cnstr & 1.404 & 1.380 & 0.373 & 0.598 & 3.356 \\
\hline
\end{tabular}

Return on Assets

\begin{tabular}{lccccc}
\hline Industry & Mean & Median & Std Dev & Min & Max \\
\hline NoDur & 0.008 & 0.008 & 0.005 & -0.014 & 0.019 \\
Utils & 0.009 & 0.008 & 0.004 & 0.002 & 0.025 \\
Hlth & -0.012 & -0.019 & 0.019 & -0.040 & 0.030 \\
Durbl & 0.005 & 0.004 & 0.008 & -0.029 & 0.021 \\
Manuf & 0.006 & 0.006 & 0.007 & -0.034 & 0.022 \\
Chems & 0.008 & 0.007 & 0.007 & -0.013 & 0.023 \\
Shops & 0.003 & 0.002 & 0.007 & -0.016 & 0.017 \\
Mines & -0.001 & -0.004 & 0.013 & -0.033 & 0.031 \\
Cnstr & 0.004 & 0.004 & 0.007 & -0.032 & 0.024 \\
\hline & & & & \\
& & Leverage Ratio & & Max \\
\hline Industry & Mean & Median & Std Dev & 0.404 & 0.595 \\
NoDur & 0.477 & 0.474 & 0.022 & 0.606 & 0.796 \\
Utils & 0.646 & 0.643 & 0.024 & 0.275 & 0.636 \\
Hlth & 0.358 & 0.353 & 0.048 & 0.407 & 0.737 \\
Durbl & 0.497 & 0.493 & 0.033 & 0.420 & 0.586 \\
Manuf & 0.490 & 0.485 & 0.031 & 0.370 & 0.591 \\
Chems & 0.491 & 0.488 & 0.045 & 0.466 & 0.775 \\
Shops & 0.512 & 0.515 & 0.025 & 0.185 & \\
Mines & 0.323 & 0.314 & 0.059 & 0.468 & \\
Cnstr & 0.562 & 0.553 & 0.043 & & \\
\hline
\end{tabular}


Panel B: Quarterly return, market to book ratio, return on assets, and leverage ratio of cyclical and non-cyclical industry portfolios

Quarterly Return

\begin{tabular}{lccccc}
\hline & Mean & Median & Std Dev & Min & Max \\
\hline 1961Q2 - 2014Q4 & & & & & \\
$\begin{array}{l}\text { Non-cyclical } \\
\text { Cyclical }\end{array}$ & 0.038 & 0.035 & 0.116 & -0.346 & 0.565 \\
$\begin{array}{l}\text { 1961Q2 - 1982Q4 } \\
\text { Non-cyclical }\end{array}$ & 0.038 & 0.034 & 0.142 & -0.408 & 0.884 \\
$\begin{array}{l}\text { Cyclical } \\
\text { 1983Q1 - 2001Q4 }\end{array}$ & 0.035 & 0.035 & 0.116 & -0.287 & 0.503 \\
$\begin{array}{l}\text { Non-cyclical } \\
\text { Cyclical }\end{array}$ & 0.042 & 0.039 & 0.147 & -0.327 & 0.884 \\
$\begin{array}{l}\text { 2002Q1 - 2014Q4 } \\
\text { Non-cyclical }\end{array}$ & 0.042 & 0.034 & 0.116 & -0.346 & 0.565 \\
Cyclical & 0.030 & 0.027 & 0.129 & -0.377 & 0.772 \\
\end{tabular}

Market to Book Ratio

\begin{tabular}{|c|c|c|c|c|c|}
\hline & Mean & Median & Std Dev & Min & Max \\
\hline \multicolumn{6}{|l|}{ 1961Q2 - 2014Q4 } \\
\hline Non-cyclical & 1.785 & 1.679 & 0.667 & 0.544 & 4.588 \\
\hline Cyclical & 1.638 & 1.672 & 0.439 & 0.409 & 3.356 \\
\hline \multicolumn{6}{|l|}{ 1961Q2 - 1982Q4 } \\
\hline Non-cyclical & 1.349 & 1.073 & 0.680 & 0.544 & 4.588 \\
\hline Cyclical & 1.184 & 1.119 & 0.361 & 0.409 & 3.356 \\
\hline \multicolumn{6}{|l|}{ 1983Q1 - 2001Q4 } \\
\hline Non-cyclical & 1.913 & 1.823 & 0.619 & 0.804 & 3.128 \\
\hline Cyclical & 1.746 & 1.745 & 0.324 & 0.909 & 2.769 \\
\hline \multicolumn{6}{|l|}{ 2002Q1 - 2014Q4 } \\
\hline Non-cyclical & 1.982 & 1.794 & 0.543 & 1.116 & 3.085 \\
\hline Cyclical & 1.883 & 1.870 & 0.341 & 1.033 & 2.923 \\
\hline \multicolumn{6}{|c|}{ Return on Assets } \\
\hline & Mean & Median & Std Dev & Min & Max \\
\hline \multicolumn{6}{|l|}{ 1961Q2 - 2014Q4 } \\
\hline Non-cyclical & 0.001 & 0.006 & 0.015 & -0.040 & 0.030 \\
\hline Cyclical & 0.004 & 0.004 & 0.009 & -0.034 & 0.031 \\
\hline \multicolumn{6}{|l|}{ 1961Q2 - 1982Q4 } \\
\hline Non-cyclical & 0.013 & 0.014 & 0.006 & -0.009 & 0.030 \\
\hline Cyclical & 0.012 & 0.014 & 0.008 & -0.034 & 0.031 \\
\hline \multicolumn{6}{|l|}{ 1983Q1 - 2001Q4 } \\
\hline Non-cyclical & -0.002 & 0.004 & 0.013 & -0.031 & 0.020 \\
\hline Cyclical & 0.001 & 0.002 & 0.007 & -0.028 & 0.014 \\
\hline \multicolumn{6}{|l|}{ 2002Q1 - 2014Q4 } \\
\hline Non-cyclical & -0.005 & 0.005 & 0.017 & -0.040 & 0.013 \\
\hline Cyclical & 0.001 & 0.003 & 0.007 & -0.033 & 0.019 \\
\hline
\end{tabular}




\section{Leverage Ratio}

\begin{tabular}{lccccc}
\hline & Mean & Median & Std Dev & Min & Max \\
\hline 1961Q2 - 2014Q4 & & & & & \\
Non-cyclical & 0.492 & 0.474 & 0.122 & 0.275 & 0.719 \\
$\begin{array}{l}\text { Cyclical } \\
\text { 1961Q2 - 1982Q4 }\end{array}$ & 0.480 & 0.494 & 0.084 & 0.185 & 0.856 \\
$\begin{array}{l}\text { Non-cyclical } \\
\text { Cyclical }\end{array}$ & 0.504 & 0.483 & 0.100 & 0.309 & 0.719 \\
1983Q1 - 2001Q4 & 0.481 & 0.486 & 0.096 & 0.185 & 0.856 \\
Non-cyclical & & & & & \\
Cyclical & 0.486 & 0.476 & 0.129 & 0.275 & 0.691 \\
2002Q1 - 2014Q4 & 0.485 & 0.500 & 0.075 & 0.260 & 0.638 \\
$\begin{array}{l}\text { Non-cyclical } \\
\text { Cyclical }\end{array}$ & 0.489 & 0.465 & 0.130 & 0.288 & 0.703 \\
\hline
\end{tabular}


Table 5

Fisher Effect

$\mathrm{R}_{\mathrm{i}, \mathrm{t}}=\beta_{0}+\beta_{1} \mathrm{E}_{\mathrm{t}-1} \pi_{\mathrm{t}}+\varepsilon_{\mathrm{i}, \mathrm{t}}$

where $\mathrm{R}_{\mathrm{i}, \mathrm{t}}$ is the average quarterly return, $\pi_{\mathrm{t}}$ is the expected quarterly inflation rate, and $\varepsilon_{\mathrm{i}, \mathrm{t}}$ is a residual and distributed as a normal distribution. Variance is estimated with the Huber/White/sandwich robust variances estimator (White, 1980). The residual of the cyclical- and non-cyclical industry portfolio is estimated based on the fixed-effects model in panel regression.

\begin{tabular}{|c|c|c|c|c|c|c|c|c|c|c|c|c|}
\hline & \multicolumn{3}{|c|}{ 1961Q2-2014Q4 } & \multicolumn{2}{|c|}{ 1961Q2-1982Q4 } & \multicolumn{4}{|c|}{ 1983Q1 - 2001Q4 } & \multicolumn{2}{|c|}{ 2002Q1 - 2014Q4 } & \multirow[b]{2}{*}{$\mathbf{R}^{2}$} \\
\hline Industry & $\beta_{1}$ & $\beta_{0}$ & $\mathbf{R}^{2}$ & $\beta_{1}$ & $\beta_{0}$ & $\mathbf{R}^{2}$ & $\beta_{1}$ & $\beta_{0}$ & $\mathbf{R}^{2}$ & $\beta_{1}$ & $\beta_{0}$ & \\
\hline NoDur & $\begin{array}{c}1.506 \\
(1.238)\end{array}$ & $\begin{array}{c}0.026 \\
(0.010)^{* * *}\end{array}$ & 0.009 & $\begin{array}{c}1.397 \\
(1.424)\end{array}$ & $\begin{array}{c}0.025 \\
(0.017)\end{array}$ & 0.010 & $\begin{array}{c}4.745 \\
(4.523)\end{array}$ & $\begin{array}{c}0.007 \\
(0.027)\end{array}$ & 0.022 & $\begin{array}{c}1.217 \\
(4.437)\end{array}$ & $\begin{array}{c}0.033 \\
(0.019)^{*}\end{array}$ & 0.003 \\
\hline Utils & $\begin{array}{c}0.280 \\
(0.922)\end{array}$ & $\begin{array}{c}0.029 \\
(0.007)^{* * *}\end{array}$ & 0.001 & $\begin{array}{c}1.068 \\
(1.134)\end{array}$ & $\begin{array}{c}0.010 \\
(0.012)\end{array}$ & 0.016 & $\begin{array}{c}0.172 \\
(1.791)\end{array}$ & $\begin{array}{c}0.039 \\
(0.013)^{* * *}\end{array}$ & 0.000 & $\begin{array}{l}-0.251 \\
(2.799)\end{array}$ & $\begin{array}{c}0.035 \\
(0.013)^{* * *}\end{array}$ & 0.000 \\
\hline Hlth & $\begin{array}{c}1.351 \\
(1.407)\end{array}$ & $\begin{array}{c}0.036 \\
(0.013)^{* * *}\end{array}$ & 0.005 & $\begin{array}{c}1.369 \\
(1.566)\end{array}$ & $\begin{array}{c}0.028 \\
(0.019)\end{array}$ & 0.010 & $\begin{array}{c}8.915 \\
(6.922)\end{array}$ & $\begin{array}{c}0.001 \\
(0.043)\end{array}$ & 0.034 & $\begin{array}{l}-1.272 \\
(5.758)\end{array}$ & $\begin{array}{c}0.047 \\
(0.021)^{* *}\end{array}$ & 0.002 \\
\hline Durbl & $\begin{array}{c}1.017 \\
(1.393)\end{array}$ & $\begin{array}{c}0.032 \\
(0.012)^{* * *}\end{array}$ & 0.003 & $\begin{array}{c}0.513 \\
(1.516)\end{array}$ & $\begin{array}{c}0.036 \\
(0.020)^{*}\end{array}$ & 0.001 & $\begin{array}{c}5.749 \\
(5.598)\end{array}$ & $\begin{array}{c}0.004 \\
(0.033)\end{array}$ & 0.020 & $\begin{array}{c}1.312 \\
(7.092)\end{array}$ & $\begin{array}{c}0.033 \\
(0.027)\end{array}$ & 0.001 \\
\hline Manuf & $\begin{array}{c}0.771 \\
(1.262)\end{array}$ & $\begin{array}{c}0.032 \\
(0.012)^{* * *}\end{array}$ & 0.002 & $\begin{array}{c}0.890 \\
(1.407)\end{array}$ & $\begin{array}{c}0.027 \\
(0.018)\end{array}$ & 0.004 & $\begin{array}{c}3.366 \\
(4.420)\end{array}$ & $\begin{array}{c}0.014 \\
(0.029)\end{array}$ & 0.010 & $\begin{array}{c}0.079 \\
(5.422)\end{array}$ & $\begin{array}{c}0.045 \\
(0.025)^{*}\end{array}$ & 0.000 \\
\hline Chems & $\begin{array}{c}0.659 \\
(1.125)\end{array}$ & $\begin{array}{c}0.033 \\
(0.011)^{* * *}\end{array}$ & 0.002 & $\begin{array}{c}0.745 \\
(1.251)\end{array}$ & $\begin{array}{c}0.027 \\
(0.017)\end{array}$ & 0.004 & $\begin{array}{c}4.490 \\
(3.797)\end{array}$ & $\begin{array}{c}0.012 \\
(0.027)\end{array}$ & 0.022 & $\begin{array}{l}-0.180 \\
(4.837)\end{array}$ & $\begin{array}{c}0.044 \\
(0.025)^{*}\end{array}$ & 0.000 \\
\hline Shops & $\begin{array}{c}1.232 \\
(1.407)\end{array}$ & $\begin{array}{c}0.029 \\
(0.012)^{* * *}\end{array}$ & 0.004 & $\begin{array}{c}1.131 \\
(1.612)\end{array}$ & $\begin{array}{c}0.031 \\
(0.020)\end{array}$ & 0.005 & $\begin{array}{c}4.177 \\
(4.802)\end{array}$ & $\begin{array}{c}0.005 \\
(0.031)\end{array}$ & 0.013 & $\begin{array}{l}-0.273 \\
(5.418)\end{array}$ & $\begin{array}{c}0.042 \\
(0.021)^{* *}\end{array}$ & 0.000 \\
\hline Mines & $\begin{array}{c}-0.882 \\
(1.749)\end{array}$ & $\begin{array}{c}0.044 \\
(0.017)^{* * *}\end{array}$ & 0.002 & $\begin{array}{c}-0.596 \\
(1.891)\end{array}$ & $\begin{array}{c}0.056 \\
(0.021)^{* * *}\end{array}$ & 0.002 & $\begin{array}{l}-3.840 \\
(6.265)\end{array}$ & $\begin{array}{c}0.037 \\
(0.046)\end{array}$ & 0.007 & $\begin{array}{l}-5.896 \\
(6.612)\end{array}$ & $\begin{array}{c}0.075 \\
(0.035)^{* *}\end{array}$ & 0.023 \\
\hline Cnstr & $\begin{array}{c}2.069 \\
(1.831)\end{array}$ & $\begin{array}{c}0.023 \\
(0.014)\end{array}$ & 0.008 & $\begin{array}{c}1.874 \\
(2.177)\end{array}$ & $\begin{array}{c}0.028 \\
(0.025)\end{array}$ & 0.008 & $\begin{array}{c}7.178 \\
(6.708)\end{array}$ & $\begin{array}{c}-0.012 \\
(0.038)\end{array}$ & 0.027 & $\begin{array}{l}-1.192 \\
(4.225)\end{array}$ & $\begin{array}{c}0.040 \\
(0.023)^{*}\end{array}$ & 0.002 \\
\hline Non-cyclical & $\begin{array}{c}1.048 \\
(0.633)^{*}\end{array}$ & $\begin{array}{c}0.030 \\
(0.007)^{* * *}\end{array}$ & 0.004 & $\begin{array}{c}1.280 \\
(0.780)^{*}\end{array}$ & $\begin{array}{c}0.021 \\
(0.011)^{*}\end{array}$ & 0.010 & $\begin{array}{c}4.611 \\
(2.322)^{* *}\end{array}$ & $\begin{array}{c}0.015 \\
(0.015)\end{array}$ & 0.017 & $\begin{array}{c}-0.102 \\
(1.969)\end{array}$ & $\begin{array}{c}0.038 \\
(0.013)^{* * *}\end{array}$ & 0.000 \\
\hline Cyclical & $\begin{array}{c}0.812 \\
(0.548)\end{array}$ & $\begin{array}{c}0.032 \\
(0.006)^{* * *}\end{array}$ & 0.002 & $\begin{array}{c}0.761 \\
(0.702)\end{array}$ & $\begin{array}{c}0.034 \\
(0.010)^{* * *}\end{array}$ & 0.002 & $\begin{array}{c}3.520 \\
(1.826)^{*}\end{array}$ & $\begin{array}{c}0.010 \\
(0.012)\end{array}$ & 0.008 & $\begin{array}{l}-1.025 \\
(1.804)\end{array}$ & $\begin{array}{c}0.046 \\
(0.011)^{* * *}\end{array}$ & 0.001 \\
\hline
\end{tabular}

. Significant at $10 \%$

**: Significant at 5\%

***: Significant at $1 \%$ 
Table 6

Inflation, M/B, ROA, and Leverage, 1961Q2 - 2014Q4

$\mathrm{M} / \mathrm{B}_{\mathrm{i}, \mathrm{t}}=\beta_{0}+\beta_{1} \mathrm{U}_{\mathrm{t}} \pi_{\mathrm{t}}+\beta_{2} \mathrm{E}_{\mathrm{t}-\mathrm{1}} \pi_{\mathrm{t}}+\varepsilon_{\mathrm{i}, \mathrm{t}}$

$\mathrm{ROA}_{\mathrm{i}, \mathrm{t}}=\beta_{0}+\beta_{1} \mathrm{U}_{\mathrm{t}} \pi_{\mathrm{t}}+\beta_{2} \mathrm{E}_{\mathrm{t}-1} \pi_{\mathrm{t}}+\varepsilon_{\mathrm{i}, \mathrm{t}}$

$\mathrm{LEV}_{\mathrm{i}, \mathrm{t}}=\beta_{0}+\beta_{1} \mathrm{U}_{\mathrm{t}} \pi_{\mathrm{t}}+\beta_{2} \mathrm{E}_{\mathrm{t}-1} \pi_{\mathrm{t}}+\varepsilon_{\mathrm{i}, \mathrm{t}}$

where $U_{t} \pi_{t}$ is the unexpected quarterly inflation rate, $\pi_{t}$ is the expected quarterly inflation rate, $M / B_{i, t}$ is the average quarterly market to book ratio, $\mathrm{ROA}_{i, t}$ is the average quarterly return on assets, $\mathrm{LEV}_{\mathrm{i}, \mathrm{t}}$ is the average quarterly leverage ratio, and $\varepsilon_{\mathrm{i}, \mathrm{t}}$ is a residual. Variance is estimated with the Huber/White/sandwich robust variances estimator (White, 1980).

\begin{tabular}{|c|c|c|c|c|c|c|c|c|c|c|c|c|}
\hline \multirow{2}{*}{$\begin{array}{r}\text { Panel A } \\
\text { Industry }\end{array}$} & \multicolumn{3}{|c|}{$\mathbf{M} / \mathbf{B}$} & \multicolumn{5}{|c|}{ ROA } & \multicolumn{4}{|c|}{ LEV } \\
\hline & $\beta_{1}$ & $\boldsymbol{\beta}_{2}$ & $\boldsymbol{\beta}_{0}$ & $\mathbf{R}^{2}$ & $\beta_{1}$ & $\beta_{2}$ & $\boldsymbol{\beta}_{0}$ & $\mathbf{R}^{2}$ & $\beta_{1}$ & $\boldsymbol{\beta}_{2}$ & $\boldsymbol{\beta}_{0}$ & $\mathbf{R}^{2}$ \\
\hline NoDur & $\begin{array}{l}-11.296 \\
(6.264)^{*}\end{array}$ & $\begin{array}{c}-38.146 \\
(3.179) * * *\end{array}$ & $\begin{array}{c}1.930 \\
(0.039)^{* * *}\end{array}$ & 0.47 & $\begin{array}{c}0.282 \\
(0.086)^{* * *}\end{array}$ & $\begin{array}{c}0.273 \\
(0.043)^{* * *}\end{array}$ & $\begin{array}{c}0.005 \\
(0.001)^{* * *}\end{array}$ & 0.21 & $\begin{array}{c}0.186 \\
(0.324)\end{array}$ & $\begin{array}{c}0.854 \\
(0.223) * * *\end{array}$ & $\begin{array}{c}0.470 \\
(0.002)^{* * *}\end{array}$ & 0.08 \\
\hline Utils & $\begin{array}{l}-3.565 \\
(3.700)\end{array}$ & $\begin{array}{c}-24.341 \\
(1.853)^{* * *}\end{array}$ & $\begin{array}{c}1.442 \\
(0.024)^{* * *}\end{array}$ & 0.42 & $\begin{array}{c}0.112 \\
(0.048)^{* *}\end{array}$ & $\begin{array}{c}0.219 \\
(0.041)^{* * *}\end{array}$ & $\begin{array}{c}0.007 \\
(0.000)^{* * *}\end{array}$ & 0.17 & $\begin{array}{l}-0.493 \\
(0.329)\end{array}$ & $\begin{array}{l}-0.423 \\
(0.277)\end{array}$ & $\begin{array}{c}0.650 \\
(0.003)^{* * *}\end{array}$ & 0.03 \\
\hline Hlth & $\begin{array}{l}-17.391 \\
(9.931)^{*}\end{array}$ & $\begin{array}{c}-35.274 \\
(3.995)^{* * *}\end{array}$ & $\begin{array}{c}2.828 \\
(0.054)^{* * *}\end{array}$ & 0.31 & $\begin{array}{c}0.670 \\
(0.191)^{* * *}\end{array}$ & $\begin{array}{c}1.805 \\
(0.106)^{* * *}\end{array}$ & $\begin{array}{c}-0.028 \\
(0.001)^{* * *}\end{array}$ & 0.50 & $\begin{array}{c}1.027 \\
(0.843)\end{array}$ & $\begin{array}{c}3.752 \\
(0.395)^{* * *}\end{array}$ & $\begin{array}{c}0.325 \\
(0.006)^{* * *}\end{array}$ & 0.33 \\
\hline Durbl & $\begin{array}{l}-6.698 \\
(7.211)\end{array}$ & $\begin{array}{c}-38.202 \\
(3.607)^{* * *}\end{array}$ & $\begin{array}{c}1.907 \\
(0.045)^{* * *}\end{array}$ & 0.48 & $\begin{array}{c}0.556 \\
(0.120)^{* * *}\end{array}$ & $\begin{array}{c}0.316 \\
(0.089)^{* * *}\end{array}$ & $\begin{array}{c}0.001 \\
(0.001)\end{array}$ & 0.22 & $\begin{array}{l}-0.178 \\
(0.401)\end{array}$ & $\begin{array}{c}0.815 \\
(0.268)^{* * *}\end{array}$ & $\begin{array}{c}0.491 \\
(0.003)^{* * *}\end{array}$ & 0.04 \\
\hline Manuf & $\begin{array}{c}-6.178 \\
(5.661)\end{array}$ & $\begin{array}{c}-32.232 \\
(3.237)^{* * *}\end{array}$ & $\begin{array}{c}1.798 \\
(0.039)^{* * *}\end{array}$ & 0.41 & $\begin{array}{c}0.532 \\
(0.064)^{* * *}\end{array}$ & $\begin{array}{c}0.449 \\
(0.048)^{* * *}\end{array}$ & $\begin{array}{c}0.002 \\
(0.001)^{* * *}\end{array}$ & 0.33 & $\begin{array}{l}-0.173 \\
(0.284)\end{array}$ & $\begin{array}{c}0.231 \\
(0.193)\end{array}$ & $\begin{array}{c}0.489 \\
(0.003)^{* * *}\end{array}$ & 0.00 \\
\hline Chems & $\begin{array}{l}-5.089 \\
(5.684)\end{array}$ & $\begin{array}{c}-38.319 \\
(3.166)^{* * *}\end{array}$ & $\begin{array}{c}2.169 \\
(0.040)^{* * *}\end{array}$ & 0.42 & $\begin{array}{c}0.564 \\
(0.059)^{* * *}\end{array}$ & $\begin{array}{c}0.548 \\
(0.051)^{* * *}\end{array}$ & $\begin{array}{c}0.003 \\
(0.001)^{* * *}\end{array}$ & 0.45 & $\begin{array}{c}-2.364 \\
(0.473)^{* * *}\end{array}$ & $\begin{array}{c}-2.583 \\
(0.308)^{* * *}\end{array}$ & $\begin{array}{c}0.517 \\
(0.004)^{* * *}\end{array}$ & 0.23 \\
\hline Shops & $\begin{array}{l}-11.808 \\
(7.193)^{*}\end{array}$ & $\begin{array}{c}-39.226 \\
(3.547)^{* * *}\end{array}$ & $\begin{array}{c}2.064 \\
(0.044)^{* * *}\end{array}$ & 0.45 & $\begin{array}{c}0.420 \\
(0.068)^{* * *}\end{array}$ & $\begin{array}{c}0.471 \\
(0.043)^{* * *}\end{array}$ & $\begin{array}{c}-0.002 \\
(0.001)^{* * *}\end{array}$ & 0.35 & $\begin{array}{c}0.393 \\
(0.458)\end{array}$ & $\begin{array}{c}1.796 \\
(0.233)^{* * *}\end{array}$ & $\begin{array}{c}0.496 \\
(0.003)^{* * *}\end{array}$ & 0.28 \\
\hline Mines & $\begin{array}{c}3.754 \\
(6.216)\end{array}$ & $\begin{array}{c}-11.406 \\
(4.324)^{* * *}\end{array}$ & $\begin{array}{c}1.844 \\
(0.054)^{* * *}\end{array}$ & 0.04 & $\begin{array}{c}0.851 \\
(0.148)^{* * *}\end{array}$ & $\begin{array}{c}1.134 \\
(0.086)^{* * *}\end{array}$ & $\begin{array}{c}-0.012 \\
(0.001)^{* * *}\end{array}$ & 0.51 & $\begin{array}{l}-1.029 \\
(0.899)\end{array}$ & $\begin{array}{c}0.573 \\
(0.762)\end{array}$ & $\begin{array}{c}0.320 \\
(0.007)^{* * *}\end{array}$ & 0.02 \\
\hline Cnstr & $\begin{array}{r}-5.940 \\
(4.660) \\
\end{array}$ & $\begin{array}{c}-24.889 \\
(2.894)^{* * *}\end{array}$ & $\begin{array}{c}1.619 \\
(0.038)^{* * *}\end{array}$ & 0.24 & $\begin{array}{c}0.435 \\
(0.089)^{* * *}\end{array}$ & $\begin{array}{c}0.200 \\
(0.087)^{* *}\end{array}$ & $\begin{array}{c}0.001 \\
(0.001)\end{array}$ & 0.13 & $\begin{array}{c}0.901 \\
(0.501)^{*}\end{array}$ & $\begin{array}{c}2.300 \\
(0.293)^{* * *}\end{array}$ & $\begin{array}{c}0.541 \\
(0.004)^{* * *}\end{array}$ & 0.16 \\
\hline
\end{tabular}

*: Significant at $10 \%$

$* *$ : Significant at $5 \%$

$* * *$ : Significant at $1 \%$ 
Table 6

Inflation, $M / B, R O A$, and Leverage (Continues)

$\mathrm{M} / \mathrm{B}_{\mathrm{i}, \mathrm{t}}=\beta_{0}+\beta_{1} \mathrm{U}_{\mathrm{t}} \pi_{\mathrm{t}}+\beta_{2} \mathrm{E}_{\mathrm{t}-1} \pi_{\mathrm{t}}+\varepsilon_{\mathrm{i}, \mathrm{t}}$

$\mathrm{ROA}_{\mathrm{i}, \mathrm{t}}=\beta_{0}+\beta_{1} \mathrm{U}_{\mathrm{t}} \pi_{\mathrm{t}}+\beta_{2} \mathrm{E}_{\mathrm{t}-1} \pi_{\mathrm{t}}+\varepsilon_{\mathrm{i}, \mathrm{t}}$

$\mathrm{LEV}_{\mathrm{i}, \mathrm{t}}=\beta_{0}+\beta_{1} \mathrm{U}_{\mathrm{t}} \pi_{\mathrm{t}}+\beta_{2} \mathrm{E}_{\mathrm{t}-1} \pi_{\mathrm{t}}+\varepsilon_{\mathrm{i}, \mathrm{t}}$

where $U_{t} \pi_{t}$ is the unexpected quarterly inflation rate, $\pi_{t}$ is the expected quarterly inflation rate, $M / B_{i, t}$ is the average quarterly market to book ratio, $\mathrm{ROA}_{\mathrm{i}, \mathrm{t}}$ is the average quarterly return on assets, $\mathrm{LEV}_{\mathrm{i}, \mathrm{t}}$ is the average quarterly leverage ratio, and $\varepsilon_{\mathrm{i}, \mathrm{t}}$ is a residual. The residual of the cyclical- and non-cyclical industry portfolio is estimated based on the fixed-effects model in panel regression.

\begin{tabular}{|c|c|c|c|c|c|c|c|c|c|c|c|c|}
\hline \multirow{2}{*}{$\begin{array}{l}\text { Panel B } \\
\text { Industry }\end{array}$} & \multicolumn{3}{|c|}{$\mathbf{M} / \mathbf{B}$} & \multicolumn{4}{|c|}{ ROA } & \multicolumn{5}{|c|}{ LEV } \\
\hline & $\beta_{1}$ & $\beta_{2}$ & $\boldsymbol{\beta}_{0}$ & $\mathbf{R}^{2}$ & $\beta_{1}$ & $\beta_{2}$ & $\boldsymbol{\beta}_{0}$ & $\mathbf{R}^{2}$ & $\beta_{1}$ & $\boldsymbol{\beta}_{2}$ & $\boldsymbol{\beta}_{0}$ & $\mathbf{R}^{2}$ \\
\hline 61Q2-14Q4 & & & & & & & & & & & & \\
\hline Non-cyclical & $\begin{array}{c}-10.829 \\
(2.532)^{* * *}\end{array}$ & $\begin{array}{c}-32.597 \\
(1.883)^{* * *}\end{array}$ & $\begin{array}{c}2.074 \\
(0.022)^{* * *}\end{array}$ & 0.13 & $\begin{array}{c}0.354 \\
(0.081)^{* * *}\end{array}$ & $\begin{array}{c}0.767 \\
(0.060)^{* * *}\end{array}$ & $\begin{array}{c}-0.006 \\
(0.001)^{* * *}\end{array}$ & 0.14 & $\begin{array}{c}0.241 \\
(0.255)\end{array}$ & $\begin{array}{c}1.399 \\
(0.190)^{* * *}\end{array}$ & $\begin{array}{c}0.480 \\
(0.002)^{* * *}\end{array}$ & 0.01 \\
\hline Cyclical & $\begin{array}{c}-5.403 \\
(1.947)^{* * *} \\
\end{array}$ & $\begin{array}{c}-30.856 \\
(1.452)^{* * *} \\
\end{array}$ & $\begin{array}{c}1.900 \\
(0.017)^{* * *} \\
\end{array}$ & 0.27 & $\begin{array}{c}0.557 \\
(0.039)^{* * *} \\
\end{array}$ & $\begin{array}{c}0.515 \\
(0.029)^{* * *} \\
\end{array}$ & $\begin{array}{c}-0.001 \\
(0.000)^{* * *} \\
\end{array}$ & 0.27 & $\begin{array}{c}-0.406 \\
(0.228)^{*}\end{array}$ & $\begin{array}{c}0.522 \\
(0.170)^{* * *} \\
\end{array}$ & $\begin{array}{c}0.476 \\
(0.002)^{* * *} \\
\end{array}$ & 0.00 \\
\hline 61Q2-82Q4 & & & & & & & & & & & & \\
\hline Non-cyclical & $\begin{array}{c}-20.177 \\
(5.180)^{* * *}\end{array}$ & $\begin{array}{c}-23.455 \\
(4.325)^{* * *}\end{array}$ & $\begin{array}{c}1.794 \\
(0.085)^{* * *}\end{array}$ & 0.10 & $\begin{array}{c}0.105 \\
(0.078)\end{array}$ & $\begin{array}{c}0.052 \\
(0.066)\end{array}$ & $\begin{array}{c}0.012 \\
(0.001)^{* * *}\end{array}$ & 0.01 & $\begin{array}{c}1.162 \\
(0.432)^{* * *}\end{array}$ & $\begin{array}{c}1.527 \\
(0.361)^{* * *}\end{array}$ & $\begin{array}{c}0.476 \\
(0.007)^{* * *}\end{array}$ & 0.03 \\
\hline Cyclical & $\begin{array}{c}-13.049 \\
(3.075)^{* * *}\end{array}$ & $\begin{array}{c}-14.727 \\
(2.528)^{* * *}\end{array}$ & $\begin{array}{c}1.462 \\
(0.049)^{* * *}\end{array}$ & 0.11 & $\begin{array}{c}0.512 \\
(0.068)^{* * *}\end{array}$ & $\begin{array}{c}0.131 \\
(0.056)^{* *}\end{array}$ & $\begin{array}{c}0.009 \\
(0.001)^{* * *}\end{array}$ & 0.16 & $\begin{array}{c}0.861 \\
(0.470)^{*}\end{array}$ & $\begin{array}{c}1.404 \\
(0.386)^{* * *}\end{array}$ & $\begin{array}{c}0.455 \\
(0.008)^{* * *}\end{array}$ & 0.01 \\
\hline 83Q1-01Q4 & & & & & & & & & & & & \\
\hline Non-cyclical & $\begin{array}{c}-8.935 \\
(3.863)^{* *}\end{array}$ & $\begin{array}{c}-12.174 \\
(4.317)^{* * *}\end{array}$ & $\begin{array}{c}2.001 \\
(0.032)^{* * *}\end{array}$ & 0.00 & $\begin{array}{c}0.217 \\
(0.107)^{* *}\end{array}$ & $\begin{array}{c}0.407 \\
(0.119)^{* * *}\end{array}$ & $\begin{array}{c}-0.005 \\
(0.001)^{* * *}\end{array}$ & 0.01 & $\begin{array}{c}0.439 \\
(0.487)\end{array}$ & $\begin{array}{c}1.225 \\
(0.545)^{* *}\end{array}$ & $\begin{array}{c}0.479 \\
(0.004)^{* * *}\end{array}$ & 0.00 \\
\hline Cyclical & $\begin{array}{c}1.656 \\
(3.536) \\
\end{array}$ & $\begin{array}{c}-14.949 \\
(3.951)^{* * *}\end{array}$ & $\begin{array}{c}1.827 \\
(0.029)^{* * *}\end{array}$ & 0.03 & $\begin{array}{c}0.308 \\
(0.073)^{* * *}\end{array}$ & $\begin{array}{l}-0.013 \\
(0.082) \\
\end{array}$ & $\begin{array}{c}0.000 \\
(0.001) \\
\end{array}$ & 0.03 & $\begin{array}{c}-0.331 \\
(0.435) \\
\end{array}$ & $\begin{array}{c}2.334 \\
(0.487)^{* * *} \\
\end{array}$ & $\begin{array}{c}0.473 \\
(0.004)^{* * *} \\
\end{array}$ & 0.01 \\
\hline 02Q1-14Q4 & & & & & & & & & & & & \\
\hline Non-cyclical & $\begin{array}{c}8.644 \\
(2.614)^{* * *}\end{array}$ & $\begin{array}{c}1.083 \\
(3.592)\end{array}$ & $\begin{array}{c}1.966 \\
(0.023)^{* * *}\end{array}$ & 0.01 & $\begin{array}{c}0.182 \\
(0.049)^{* * *}\end{array}$ & $\begin{array}{c}0.038 \\
(0.067)\end{array}$ & $\begin{array}{c}-0.005 \\
(0.000)^{* * *}\end{array}$ & 0.00 & $\begin{array}{c}-0.765 \\
(0.312)^{* *}\end{array}$ & $\begin{array}{l}-0.017 \\
(0.429)\end{array}$ & $\begin{array}{c}0.490 \\
(0.003)^{* * *}\end{array}$ & 0.00 \\
\hline Cyclical & $\begin{array}{c}13.584 \\
(2.541)^{* * *}\end{array}$ & $\begin{array}{r}2.635 \\
(3.491) \\
\end{array}$ & $\begin{array}{c}1.854 \\
(0.023)^{* * *}\end{array}$ & 0.07 & $\begin{array}{c}0.423 \\
(0.048)^{* * *}\end{array}$ & $\begin{array}{c}0.186 \\
(0.065)^{* * *}\end{array}$ & $\begin{array}{c}0.000 \\
(0.000) \\
\end{array}$ & 0.14 & $\begin{array}{c}-1.072 \\
(0.196)^{* * *}\end{array}$ & $\begin{array}{l}-0.164 \\
(0.270) \\
\end{array}$ & $\begin{array}{c}0.473 \\
(0.002)^{* * *}\end{array}$ & 0.01 \\
\hline
\end{tabular}

*: Significant at $10 \%$

**: Significant at 5\%

$* * *$ : Significant at $1 \%$ 
Table 7

Fisher Effect with M/B, ROA, and Leverage, 1961Q2 - 2014Q4

$\mathrm{R}_{\mathrm{i}, \mathrm{t}}=\beta_{0}+\beta_{1} \mathrm{U}_{\mathrm{t}} \pi_{\mathrm{t}}+\beta_{2} \mathrm{E}_{\mathrm{t}-1} \pi_{\mathrm{t}}+\beta_{3} \mathrm{M} / \mathrm{B}_{\mathrm{i}, \mathrm{t}}+\beta_{4} \mathrm{ROA}_{\mathrm{i}, \mathrm{t}}+\beta_{5} \mathrm{LEV}_{\mathrm{i}, \mathrm{t}}+\varepsilon_{\mathrm{i}, \mathrm{t}}$

where $R_{i, t}$ is the average quarterly return, $U_{t} \pi_{t}$ is the unexpected quarterly inflation rate, $\pi_{t}$ is the expected quarterly inflation rate, M/ $B_{i, t}$ is the average quarterly market to book ratio, $\mathrm{ROA}_{\mathrm{i}, \mathrm{t}}$ is the average quarterly return on assets, $\mathrm{LEV}_{\mathrm{i}, \mathrm{t}}$ is the average quarterly leverage ratio, and $\varepsilon_{\mathrm{i}, \mathrm{t}}$ is a residual. Variance is estimated with the Huber/White/sandwich robust variances estimator (White, 1980).

\begin{tabular}{|c|c|c|c|c|c|c|c|}
\hline Industry & $\beta_{1}$ & $\boldsymbol{\beta}_{2}$ & $\boldsymbol{\beta}_{3}$ & $\beta_{4}$ & $\beta_{5}$ & $\boldsymbol{\beta}_{0}$ & $\mathbf{R}^{2}$ \\
\hline \multirow[t]{2}{*}{ NoDur } & -3.735 & 3.241 & 0.078 & 4.047 & -0.173 & -0.054 & 0.089 \\
\hline & $(2.578)$ & (1.711) & $(0.039)^{* *}$ & (2.623) & $(0.485)$ & $(0.271)$ & \\
\hline \multirow[t]{2}{*}{ Utils } & -1.078 & 0.507 & 0.033 & -0.484 & -0.715 & 0.459 & 0.052 \\
\hline & (1.669) & (1.267) & $(0.031)$ & (1.530) & $(0.315)^{* *}$ & $(0.183)^{* * *}$ & \\
\hline \multirow[t]{2}{*}{ Hlth } & -2.623 & 3.269 & 0.168 & 0.137 & 0.919 & -0.722 & 0.148 \\
\hline & (3.159) & $(2.334)$ & $(0.040)^{* * *}$ & $(0.939)$ & $(0.356)^{* * *}$ & $(0.208)^{* * *}$ & \\
\hline \multirow[t]{2}{*}{ Durbl } & -3.687 & 4.793 & 0.107 & 0.363 & 0.101 & -0.215 & 0.070 \\
\hline & $(3.500)$ & $(2.366)^{* *}$ & $(0.053)^{* *}$ & (3.203) & $(0.553)$ & $(0.349)$ & \\
\hline \multirow[t]{2}{*}{ Manuf } & -0.627 & 4.527 & 0.102 & -0.942 & -0.342 & 0.020 & 0.066 \\
\hline & $(3.288)$ & $(2.338)^{* *}$ & $(0.036)^{* * *}$ & $(2.350)$ & $(0.374)$ & $(0.190)$ & \\
\hline \multirow[t]{2}{*}{ Chems } & -2.219 & 1.752 & 0.060 & 0.725 & -0.266 & 0.043 & 0.042 \\
\hline & $(3.180)$ & (1.965) & $(0.026)^{* *}$ & $(2.112)$ & $(0.275)$ & $(0.150)$ & \\
\hline \multirow[t]{2}{*}{ Shops } & -4.353 & 2.492 & 0.079 & 3.634 & 0.002 & -0.123 & 0.071 \\
\hline & (3.334) & $(2.067)$ & $(0.041)^{*}$ & $(1.892)^{*}$ & $(0.468)$ & $(0.266)$ & \\
\hline \multirow[t]{2}{*}{ Mines } & -3.114 & -5.715 & 0.171 & 6.710 & 0.946 & -0.511 & 0.251 \\
\hline & $(2.700)$ & $(2.176)^{* * *}$ & $(0.028)^{* * *}$ & $(1.146)^{* * *}$ & $(0.157)^{* * *}$ & $(0.080)^{* * *}$ & \\
\hline \multirow[t]{2}{*}{ Cnstr } & -3.900 & 4.674 & 0.092 & 0.959 & -0.329 & 0.060 & 0.080 \\
\hline & $(3.976)$ & $(2.114)^{* *}$ & $(0.047)^{* *}$ & $(1.788)$ & $(0.241)$ & $(0.171)$ & \\
\hline
\end{tabular}

*: Significant at $10 \%$

$* *$ : Significant at $5 \%$

$* * *$ : Significant at $1 \%$ 
Table 7

Fisher Effect with M/B, ROA, and Leverage (Continues)

$\mathrm{R}_{\mathrm{i}, \mathrm{t}}=\beta_{0}+\beta_{1} \mathrm{U}_{\mathrm{t}} \pi_{\mathrm{t}}+\beta_{2} \mathrm{E}_{\mathrm{t}-1} \pi_{\mathrm{t}}+\beta_{3} \mathrm{M} / \mathrm{B}_{\mathrm{i}, \mathrm{t}}+\beta_{4} \mathrm{ROA}_{\mathrm{i}, \mathrm{t}}+\beta_{5} \mathrm{LEV}_{\mathrm{i}, \mathrm{t}}+\varepsilon_{\mathrm{i}, \mathrm{t}}$

where $R_{i, t}$ is the average quarterly return, $U_{t} \pi_{t}$ is the unexpected quarterly inflation rate, $\pi_{t}$ is the expected quarterly inflation rate, $M / B_{i, t}$ is the average quarterly market to book ratio, $\mathrm{ROA}_{\mathrm{i}, \mathrm{t}}$ is the average quarterly return on assets, $\mathrm{LEV}_{\mathrm{i}, \mathrm{t}}$ is the average quarterly leverage ratio, and $\varepsilon_{i, t}$ is a residual. The residual of the cyclical- and non-cyclical industry portfolio is estimated based on the fixed-effects model in panel regression.

\section{Panel B}

\begin{tabular}{|c|c|c|c|c|c|c|c|}
\hline & $\beta_{1}$ & $\boldsymbol{\beta}_{2}$ & $\boldsymbol{\beta}_{3}$ & $\boldsymbol{\beta}_{4}$ & $\beta_{5}$ & $\beta_{0}$ & $\mathbf{R}^{2}$ \\
\hline \multicolumn{8}{|l|}{ 1961Q2 - 2014Q4 } \\
\hline Non-cyclical & $\begin{array}{c}-2.441 \\
(0.948)^{* * *}\end{array}$ & $\begin{array}{c}2.720 \\
(0.908)^{* * *}\end{array}$ & $\begin{array}{c}0.079 \\
(0.017)^{* * *}\end{array}$ & $\begin{array}{c}0.921 \\
(0.534)^{*}\end{array}$ & $\begin{array}{l}-0.084 \\
(0.177)\end{array}$ & $\begin{array}{l}-0.079 \\
(0.102)\end{array}$ & 0.038 \\
\hline Cyclical & $\begin{array}{c}-2.871 \\
(0.867)^{* * *}\end{array}$ & $\begin{array}{c}2.575 \\
(0.784)^{* * *}\end{array}$ & $\begin{array}{c}0.091 \\
(0.013)^{* * *}\end{array}$ & $\begin{array}{c}2.144 \\
(0.668)^{* * *}\end{array}$ & $\begin{array}{c}0.123 \\
(0.117)\end{array}$ & $\begin{array}{c}-0.196 \\
(0.065)^{* * *}\end{array}$ & 0.057 \\
\hline \multicolumn{8}{|l|}{ 1961Q2 - 1982Q4 } \\
\hline Non-cyclical & $\begin{array}{c}-7.626 \\
(1.716)^{* * *}\end{array}$ & $\begin{array}{c}0.118 \\
(1.510)\end{array}$ & $\begin{array}{c}0.013 \\
(0.029)\end{array}$ & $\begin{array}{c}-0.280 \\
(1.813)\end{array}$ & $\begin{array}{c}0.071 \\
(0.357)\end{array}$ & $\begin{array}{c}0.008 \\
(0.202)\end{array}$ & 0.174 \\
\hline Cyclical & $\begin{array}{c}-9.334 \\
(1.772)^{* * *}\end{array}$ & $\begin{array}{c}-0.678 \\
(1.363)\end{array}$ & $\begin{array}{c}0.013 \\
(0.030)\end{array}$ & $\begin{array}{c}3.198 \\
(1.486)^{* *}\end{array}$ & $\begin{array}{c}0.427 \\
(0.213)^{* *}\end{array}$ & $\begin{array}{c}-0.182 \\
(0.111)^{*}\end{array}$ & 0.101 \\
\hline \multicolumn{8}{|l|}{ 1983Q1 - 2001Q4 } \\
\hline Non-cyclical & $\begin{array}{c}-4.462 \\
(2.114)^{* *}\end{array}$ & $\begin{array}{c}4.043 \\
(2.416)^{*}\end{array}$ & $\begin{array}{c}0.217 \\
(0.036)^{* * *}\end{array}$ & $\begin{array}{c}1.932 \\
(1.338)\end{array}$ & $\begin{array}{l}-0.244 \\
(0.297)\end{array}$ & $\begin{array}{l}-0.265 \\
(0.172)\end{array}$ & 0.039 \\
\hline Cyclical & $\begin{array}{c}-6.698 \\
(1.669)^{* * *}\end{array}$ & $\begin{array}{c}3.137 \\
(1.889)^{*}\end{array}$ & $\begin{array}{c}0.198 \\
(0.025)^{* * *}\end{array}$ & $\begin{array}{c}1.136 \\
(1.216)\end{array}$ & $\begin{array}{c}0.271 \\
(0.224)\end{array}$ & $\begin{array}{c}-0.452 \\
(0.133)^{* * *}\end{array}$ & 0.134 \\
\hline \multicolumn{8}{|l|}{ 2002Q1 - 2014Q4 } \\
\hline Non-cyclical & $\begin{array}{c}1.059 \\
(1.485)\end{array}$ & $\begin{array}{c}-0.078 \\
(1.899)\end{array}$ & $\begin{array}{c}0.130 \\
(0.047)^{* * *}\end{array}$ & $\begin{array}{c}5.354 \\
(2.340)^{* *}\end{array}$ & $\begin{array}{c}0.522 \\
(0.393)\end{array}$ & $\begin{array}{c}-0.449 \\
(0.242)^{*}\end{array}$ & 0.011 \\
\hline Cyclical & $\begin{array}{c}2.631 \\
(1.451)^{*}\end{array}$ & $\begin{array}{r}-1.000 \\
(1.772) \\
\end{array}$ & $\begin{array}{c}0.115 \\
(0.036)^{* * *}\end{array}$ & $\begin{array}{c}1.818 \\
(1.691) \\
\end{array}$ & $\begin{array}{c}0.772 \\
(0.433)^{*}\end{array}$ & $\begin{array}{c}-0.540 \\
(0.243)^{* *}\end{array}$ & 0.019 \\
\hline
\end{tabular}

*: Significant at $10 \%$

**: Significant at $5 \%$

***: Significant at $1 \%$ 
Table 8

Fisher Effect with Three Factor Model, 1961Q2 - 2014 Q4

$\mathrm{R}_{\mathrm{i}, \mathrm{t}}-\mathrm{R}_{\mathrm{f}, \mathrm{t}}=\beta_{0}+\beta_{1} \mathrm{U}_{\mathrm{t}} \pi_{\mathrm{t}}+\beta_{2} \mathrm{E}_{\mathrm{t}-1} \pi_{\mathrm{t}}+\beta_{3} \mathrm{MRP}_{\mathrm{t}}+\beta_{4} \mathrm{SMB}_{\mathrm{t}}+\beta_{5} \mathrm{HML}_{\mathrm{t}}+\varepsilon_{\mathrm{i}, \mathrm{t}}$

where $R_{i, t}$ is the average quarterly return, $R_{f t}$ is the quarterly rate on one-month Treasury bill, $U_{t} \pi_{t}$ is the unexpected quarterly inflation rate, $\pi_{t}$ is the expected quarterly inflation rate, $\mathrm{MRP}_{\mathrm{t}}$ is the quarterly market risk premium, $\mathrm{SMB}_{\mathrm{t}}$ is the quarterly size premium, $\mathrm{HML}_{\mathrm{t}}$ is the quarterly value premium, and $\varepsilon_{\mathrm{i}, \mathrm{t}}$ is a residual. Variance is estimated with the Huber/White/sandwich robust variances estimator (White, 1980).

\begin{tabular}{|c|c|c|c|c|c|c|c|}
\hline Industry & $\beta_{1}$ & $\boldsymbol{\beta}_{2}$ & $\boldsymbol{\beta}_{3}$ & $\boldsymbol{\beta}_{4}$ & $\beta_{5}$ & $\boldsymbol{\beta}_{0}$ & $\mathbf{R}^{2}$ \\
\hline \multirow[t]{2}{*}{ NoDur } & -1.679 & 0.350 & 1.049 & 0.747 & 0.577 & -0.001 & 0.874 \\
\hline & $(0.546)^{* * *}$ & $(0.394)$ & $(0.060)^{* * *}$ & $(0.104)^{* * *}$ & $(0.083)^{* * *}$ & $(0.005)$ & \\
\hline \multirow[t]{2}{*}{ Utils } & -0.726 & -0.131 & 0.686 & -0.009 & 0.360 & 0.006 & 0.627 \\
\hline & $(0.473)$ & $(0.534)$ & $(0.045)^{* * *}$ & $(0.070)$ & $(0.068)^{* * *}$ & $(0.005)$ & \\
\hline \multirow[t]{2}{*}{ Hlth } & -1.512 & 0.931 & 1.291 & 0.392 & 0.057 & 0.008 & 0.705 \\
\hline & $(0.821)^{*}$ & $(0.602)$ & $(0.134)^{* * *}$ & $(0.286)$ & $(0.163)$ & $(0.007)$ & \\
\hline \multirow[t]{2}{*}{ Durbl } & -1.698 & -0.294 & 1.234 & 0.981 & 0.662 & 0.001 & 0.820 \\
\hline & $(1.016) *$ & $(0.636)$ & $(0.095)^{* * *}$ & $(0.154)^{* * *}$ & $(0.127)^{* * *}$ & $(0.007)$ & \\
\hline \multirow[t]{2}{*}{ Manuf } & 0.360 & -0.121 & 1.148 & 0.825 & 0.563 & -0.004 & 0.882 \\
\hline & $(0.496)$ & $(0.458)$ & $(0.063)^{* * *}$ & $(0.127)^{* * *}$ & $(0.087)^{* * *}$ & $(0.004)$ & \\
\hline \multirow[t]{2}{*}{ Chems } & 0.268 & 0.078 & 1.076 & 0.523 & 0.404 & 0.000 & 0.840 \\
\hline & $(0.593)$ & $(0.362)$ & $(0.046)^{* * *}$ & $(0.062)^{* * *}$ & $(0.071)^{* * *}$ & $(0.005)$ & \\
\hline \multirow[t]{2}{*}{ Shops } & -0.969 & 0.010 & 1.137 & 0.993 & 0.427 & 0.001 & 0.883 \\
\hline & $(0.545)^{*}$ & $(0.481)$ & $(0.077)^{* * *}$ & $(0.141)^{* * *}$ & $(0.106)^{* * *}$ & $(0.006)$ & \\
\hline \multirow[t]{2}{*}{ Mines } & 4.044 & -1.481 & 0.704 & 0.828 & 0.336 & 0.008 & 0.313 \\
\hline & $(1.803)^{* *}$ & (1.339) & $(0.134)^{* * *}$ & $(0.150)^{* * *}$ & $(0.169)^{* *}$ & $(0.015)$ & \\
\hline \multirow[t]{2}{*}{ Cnstr } & -1.268 & 0.676 & 1.346 & 1.141 & 0.817 & -0.013 & 0.817 \\
\hline & $(0.895)$ & $(0.736)$ & $(0.102)^{* * *}$ & $(0.174)^{* * *}$ & $(0.140)^{* * *}$ & $(0.008)$ & \\
\hline
\end{tabular}

\footnotetext{
*: Significant at $10 \%$

**: Significant at $5 \%$

$* * *$ : Significant at $1 \%$
} 
Table 8

Fisher Effect with Three Factor Model (Continues)

$\mathrm{R}_{\mathrm{i}, \mathrm{t}}-\mathrm{R}_{\mathrm{f}, \mathrm{t}}=\beta_{0}+\beta_{1} \mathrm{U}_{\mathrm{t}} \pi_{\mathrm{t}}+\beta_{2} \mathrm{E}_{\mathrm{t}-1} \pi_{\mathrm{t}}+\beta_{3} \mathrm{MRP}_{\mathrm{t}}+\beta_{4} \mathrm{SMB}_{\mathrm{t}}+\beta_{5} \mathrm{HML}_{\mathrm{t}}+\varepsilon_{\mathrm{i}, \mathrm{t}}$

where $R_{i, t}$ is the average quarterly return, $R_{f t}$ is the quarterly rate on one-month Treasury bill, $U_{t} \pi_{t}$ is the unexpected quarterly inflation rate, $\pi_{t}$ is the expected quarterly inflation rate, $\mathrm{MRP}_{t}$ is the quarterly market risk premium, $\mathrm{SMB}_{t}$ is the quarterly size premium, $\mathrm{HML}_{t}$ is the quarterly value premium, and $\varepsilon_{\mathrm{i}, \mathrm{t}}$ is a residual. The residual of the cyclical- and non-cyclical industry portfolio is estimated based on the fixed-effects model in panel regression.

\begin{tabular}{|c|c|c|c|c|c|c|c|}
\hline $\begin{array}{l}\text { anel B } \\
\text { Industry }\end{array}$ & $\beta_{1}$ & $\boldsymbol{\beta}_{2}$ & $\boldsymbol{\beta}_{3}$ & $\beta_{4}$ & $\beta_{5}$ & $\boldsymbol{\beta}_{0}$ & $\mathbf{R}^{2}$ \\
\hline \multicolumn{8}{|l|}{ 1961Q2 - 2014Q4 } \\
\hline Non-cyclical & $\begin{array}{c}-1.306 \\
(0.532)^{* * *}\end{array}$ & $\begin{array}{c}0.384 \\
(0.385)\end{array}$ & $\begin{array}{c}1.009 \\
(0.036)^{* * *}\end{array}$ & $\begin{array}{c}0.377 \\
(0.050)^{* * *}\end{array}$ & $\begin{array}{c}0.331 \\
(0.048)^{* * *}\end{array}$ & $\begin{array}{c}0.004 \\
(0.004)\end{array}$ & 0.659 \\
\hline Cyclical & $\begin{array}{c}0.121 \\
(0.429)\end{array}$ & $\begin{array}{l}-0.191 \\
(0.311)\end{array}$ & $\begin{array}{c}1.108 \\
(0.029)^{* * *}\end{array}$ & $\begin{array}{c}0.882 \\
(0.040)^{* * *}\end{array}$ & $\begin{array}{c}0.535 \\
(0.038)^{* * *}\end{array}$ & $\begin{array}{l}-0.001 \\
(0.004)\end{array}$ & 0.704 \\
\hline \multicolumn{8}{|l|}{ 1961Q2 - 1982Q4 } \\
\hline Non-cyclical & $\begin{array}{c}-1.320 \\
(0.724)^{*}\end{array}$ & $\begin{array}{c}0.203 \\
(0.401)\end{array}$ & $\begin{array}{c}0.964 \\
(0.052)^{* * *}\end{array}$ & $\begin{array}{c}0.454 \\
(0.065)^{* * *}\end{array}$ & $\begin{array}{c}0.204 \\
(0.071)^{* * *}\end{array}$ & $\begin{array}{c}0.005 \\
(0.007)\end{array}$ & 0.774 \\
\hline Cyclical & $\begin{array}{c}0.197 \\
(0.580)\end{array}$ & $\begin{array}{l}-0.372 \\
(0.321)\end{array}$ & $\begin{array}{c}1.084 \\
(0.042)^{* * *}\end{array}$ & $\begin{array}{c}1.003 \\
(0.052)^{* * *}\end{array}$ & $\begin{array}{c}0.378 \\
(0.057)^{* * *}\end{array}$ & $\begin{array}{c}0.004 \\
(0.005)\end{array}$ & 0.821 \\
\hline \multicolumn{8}{|l|}{ 1983Q1 - 2001Q4 } \\
\hline Non-cyclical & $\begin{array}{l}-2.161 \\
(1.654)\end{array}$ & $\begin{array}{c}3.614 \\
(1.794)^{* *}\end{array}$ & $\begin{array}{c}1.061 \\
(0.079)^{* * *}\end{array}$ & $\begin{array}{c}0.292 \\
(0.095)^{* * *}\end{array}$ & $\begin{array}{c}0.472 \\
(0.094)^{* * *}\end{array}$ & $\begin{array}{l}-0.018 \\
(0.014)\end{array}$ & 0.524 \\
\hline Cyclical & $\begin{array}{c}0.112 \\
(1.205)\end{array}$ & $\begin{array}{c}2.031 \\
(1.306)\end{array}$ & $\begin{array}{c}1.098 \\
(0.058)^{* * *}\end{array}$ & $\begin{array}{c}0.769 \\
(0.069)^{* * *}\end{array}$ & $\begin{array}{c}0.635 \\
(0.068)^{* * *}\end{array}$ & $\begin{array}{c}-0.025 \\
(0.010)^{* * *}\end{array}$ & 0.588 \\
\hline \multicolumn{8}{|l|}{ 2002Q1 - 2014Q4 } \\
\hline Non-cyclical & $\begin{array}{l}-0.166 \\
(0.787)\end{array}$ & $\begin{array}{c}1.728 \\
(1.165)\end{array}$ & $\begin{array}{c}1.023 \\
(0.067)^{* * *}\end{array}$ & $\begin{array}{c}0.687 \\
(0.147)^{* * *}\end{array}$ & $\begin{array}{l}-0.038 \\
(0.118)\end{array}$ & $\begin{array}{c}0.003 \\
(0.007)\end{array}$ & 0.725 \\
\hline Cyclical & $\begin{array}{c}0.468 \\
(0.778)\end{array}$ & $\begin{array}{l}-0.252 \\
(1.152)\end{array}$ & $\begin{array}{c}1.169 \\
(0.066)^{* * *}\end{array}$ & $\begin{array}{c}0.933 \\
(0.145)^{* * *}\end{array}$ & $\begin{array}{c}0.324 \\
(0.117)^{* * *}\end{array}$ & $\begin{array}{c}0.008 \\
(0.007)\end{array}$ & 0.674 \\
\hline
\end{tabular}

*: Significant at $10 \%$

**: Significant at 5\%

***: Significant at $1 \%$ 
Appendix A

Fisher Effect with Real Return

$\mathrm{r}_{\mathrm{i}, \mathrm{t}}=\beta_{0}+\beta_{1} \mathrm{E}_{\mathrm{t}-1} \pi_{\mathrm{t}}+\varepsilon_{\mathrm{i}, \mathrm{t}}$

where $\mathrm{r}_{\mathrm{i}, \mathrm{t}}$ is the average quarterly real return, $\pi_{\mathrm{t}}$ is the expected quarterly inflation rate, and $\varepsilon_{\mathrm{i}, \mathrm{t}}$ is a residual and distributed as a normal distribution. Variance is estimated with the Huber/White/sandwich robust variances estimator (White, 1980). The residual of the cyclical- and non-cyclical industry portfolio is estimated based on the fixed-effects model in panel regression.

\begin{tabular}{|c|c|c|c|c|c|c|c|c|c|c|c|c|}
\hline & \multicolumn{3}{|c|}{ 1961Q2-2014Q4 } & \multicolumn{2}{|c|}{ 1961Q2-1982Q4 } & & \multicolumn{2}{|c|}{$1983 Q 1-2001 Q 4$} & \multicolumn{4}{|c|}{ 2002Q1-2014Q4 } \\
\hline Industry & $\beta_{1}$ & $\beta_{0}$ & $\mathbf{R}^{2}$ & $\beta_{1}$ & $\beta_{0}$ & $\mathbf{R}^{2}$ & $\beta_{1}$ & $\beta_{0}$ & $\mathbf{R}^{2}$ & $\beta_{1}$ & $\boldsymbol{\beta}_{0}$ & $\mathbf{R}^{2}$ \\
\hline \multirow[t]{2}{*}{ NoDur } & 0.643 & 0.023 & 0.002 & 0.580 & 0.020 & 0.002 & 4.192 & 0.002 & 0.017 & 0.347 & 0.032 & 0.000 \\
\hline & $(1.257)$ & $(0.010)^{* *}$ & & $(1.450)$ & $(0.018)$ & & $(4.579)$ & $(0.028)$ & & $(4.488)$ & $(0.019)^{*}$ & \\
\hline \multirow[t]{2}{*}{ Utils } & -0.580 & 0.026 & 0.004 & 0.267 & 0.005 & 0.001 & -0.381 & 0.034 & 0.001 & -1.121 & 0.033 & 0.006 \\
\hline & $(0.933)$ & $(0.007)^{* * *}$ & & $(1.148)$ & $(0.012)$ & & $(1.810)$ & $(0.013)^{* * *}$ & & $(2.818)$ & $(0.013)^{* *}$ & \\
\hline \multirow[t]{2}{*}{ Hlth } & 0.489 & 0.033 & 0.001 & 0.559 & 0.023 & 0.002 & 8.363 & -0.004 & 0.029 & -2.142 & 0.045 & 0.005 \\
\hline & $(1.424)$ & $(0.013)^{* * *}$ & & (1.589) & (0.019) & & $(6.981)$ & $(0.043)$ & & $(5.808)$ & $(0.021)^{* *}$ & \\
\hline \multirow[t]{2}{*}{ Durbl } & 0.154 & 0.028 & 0.000 & -0.304 & 0.032 & 0.000 & 5.196 & -0.001 & 0.016 & 0.442 & 0.031 & 0.000 \\
\hline & $(1.414)$ & $(0.012)^{* *}$ & & $(1.546)$ & $(0.020)$ & & $(5.659)$ & $(0.033)$ & & (7.138) & $(0.027)$ & \\
\hline \multirow[t]{2}{*}{ Manuf } & -0.093 & 0.028 & 0.000 & 0.073 & 0.022 & 0.000 & 2.813 & 0.009 & 0.007 & -0.790 & 0.043 & 0.001 \\
\hline & (1.277) & $(0.012)^{* *}$ & & $(1.431)$ & (0.019) & & $(4.468)$ & $(0.029)$ & & $(5.449)$ & $(0.025)^{*}$ & \\
\hline \multirow[t]{2}{*}{ Chems } & -0.205 & 0.030 & 0.000 & -0.072 & 0.022 & 0.000 & 3.937 & 0.007 & 0.017 & -1.049 & 0.042 & 0.002 \\
\hline & (1.137) & $(0.011)^{* * *}$ & & $(1.271)$ & $(0.017)$ & & $(3.836)$ & $(0.027)$ & & $(4.860)$ & $(0.024)^{*}$ & \\
\hline \multirow[t]{2}{*}{ Shops } & 0.368 & 0.026 & 0.000 & 0.314 & 0.026 & 0.000 & 3.624 & 0.001 & 0.010 & -1.143 & 0.040 & 0.002 \\
\hline & $(1.425)$ & $(0.012)^{* *}$ & & $(1.636)$ & $(0.020)$ & & $(4.857)$ & $(0.031)$ & & $(5.466)$ & $(0.021)^{*}$ & \\
\hline \multirow[t]{2}{*}{ Mines } & -1.741 & 0.041 & 0.006 & -1.397 & 0.051 & 0.009 & -4.393 & 0.033 & 0.009 & -6.765 & 0.073 & 0.030 \\
\hline & $(1.745)$ & $(0.017)^{* *}$ & & (1.893) & $(0.021)^{* *}$ & & $(6.266)$ & $(0.046)$ & & (6.598) & $(0.034)^{* *}$ & \\
\hline \multirow[t]{2}{*}{ Cnstr } & 1.210 & 0.020 & 0.003 & 1.073 & 0.023 & 0.003 & 6.625 & -0.016 & 0.023 & -2.062 & 0.038 & 0.005 \\
\hline & $(1.847)$ & $(0.014)$ & & $(2.198)$ & $(0.026)$ & & $(6.768)$ & $(0.038)$ & & $(4.269)$ & $(0.023)^{*}$ & \\
\hline \multirow[t]{2}{*}{ Non-cyclical } & 0.186 & 0.027 & 0.000 & 0.471 & 0.016 & 0.001 & 4.058 & 0.011 & 0.013 & -0.972 & 0.037 & 0.002 \\
\hline & $(0.638)$ & $(0.007)^{* * *}$ & & $(0.794)$ & $(0.011)$ & & $(2.336)^{*}$ & $(0.015)$ & & (1.955) & $(0.012)^{* * *}$ & \\
\hline \multirow[t]{2}{*}{ Cyclical } & -0.051 & 0.029 & 0.000 & -0.051 & 0.029 & 0.000 & 2.967 & 0.005 & 0.006 & -1.895 & 0.045 & 0.004 \\
\hline & $(0.550)$ & $(0.006)^{* * *}$ & & $(0.710)$ & $(0.010)^{* * *}$ & & $(1.835)$ & $(0.012)$ & & (1.791) & $(0.011)^{* * *}$ & \\
\hline
\end{tabular}

*: Significant at $10 \%$

**: Significant at 5\%

$* * *$ : Significant at $1 \%$ 
Appendix B

Lagged Inflation, M/B, ROA, and Leverage, 1961Q2 - 2014Q4

$\mathrm{M} / \mathrm{B}_{\mathrm{i}, \mathrm{t}}=\beta_{0}+\beta_{1} \mathrm{U}_{\mathrm{t}-1} \pi_{\mathrm{t}-1}+\beta_{2} \mathrm{E}_{\mathrm{t}-2} \pi_{\mathrm{t}-1}+\varepsilon_{\mathrm{i}, \mathrm{t}}$

$\mathrm{ROA}_{\mathrm{i}, \mathrm{t}}=\beta_{0}+\beta_{1} \mathrm{U}_{\mathrm{t}-1} \pi_{\mathrm{t}-1}+\beta_{2} \mathrm{E}_{\mathrm{t}-2} \pi_{\mathrm{t}-1}+\varepsilon_{\mathrm{i}, \mathrm{t}}$

$\mathrm{LEV}_{\mathrm{i}, \mathrm{t}}=\beta_{0}+\beta_{1} \mathrm{U}_{\mathrm{t}-1} \pi_{\mathrm{t}-1}+\beta_{2} \mathrm{E}_{\mathrm{t}-2} \pi_{\mathrm{t}-1}+\varepsilon_{\mathrm{i}, \mathrm{t}}$

where $\mathrm{U}_{\mathrm{t}-1} \pi_{\mathrm{t}-1}$ is the unexpected quarterly inflation rate at $\mathrm{t}-1, \pi_{\mathrm{t}-1}$ is the expected quarterly inflation rate at $\mathrm{t}-1, \mathrm{M} / \mathrm{B}_{\mathrm{i}, \mathrm{t}}$ is the average quarterly market to book ratio, $\mathrm{ROA}_{\mathrm{i}, \mathrm{t}}$ is the average quarterly return on assets, $\mathrm{LEV}_{\mathrm{i}, \mathrm{t}}$ is the average quarterly leverage ratio, and $\varepsilon_{\mathrm{i}, \mathrm{t}}$ is a residual. Variance is estimated with the Huber/White/sandwich robust variances estimator (White, 1980).

\begin{tabular}{|c|c|c|c|c|c|c|c|c|c|c|c|c|}
\hline \multirow{2}{*}{$\begin{array}{r}\text { Panel A } \\
\text { Industry }\end{array}$} & \multicolumn{3}{|c|}{$\mathbf{M} / \mathbf{B}$} & \multicolumn{5}{|c|}{ ROA } & \multicolumn{4}{|c|}{ LEV } \\
\hline & $\beta_{1}$ & $\boldsymbol{\beta}_{2}$ & $\boldsymbol{\beta}_{0}$ & $\mathbf{R}^{2}$ & $\beta_{1}$ & $\beta_{2}$ & $\boldsymbol{\beta}_{0}$ & $\mathbf{R}^{2}$ & $\beta_{1}$ & $\beta_{2}$ & $\boldsymbol{\beta}_{0}$ & $\mathbf{R}^{2}$ \\
\hline \multirow[t]{2}{*}{ NoDur } & -13.667 & -38.019 & 1.938 & 0.47 & 0.189 & 0.268 & 0.005 & 0.16 & 0.521 & 0.692 & 0.470 & 0.06 \\
\hline & $(7.319)^{*}$ & $(3.255) * * *$ & $(0.042)^{* * *}$ & & $(0.062)^{* * *}$ & $(0.042)^{* * *}$ & $(0.000)^{* * *}$ & & $(0.393)$ & $(0.189)^{* * *}$ & $(0.002)^{* * *}$ & \\
\hline \multirow[t]{2}{*}{ Utils } & -5.823 & -25.389 & 1.458 & 0.45 & 0.099 & 0.186 & 0.007 & 0.12 & -0.497 & -0.379 & 0.650 & 0.02 \\
\hline & $(4.332)$ & $(1.862) * * *$ & $(0.026)^{* * *}$ & & $(0.051)^{*}$ & $(0.042)^{* * *}$ & $(0.000)^{* * *}$ & & $(0.318)$ & $(0.248)$ & $(0.003)^{* * *}$ & \\
\hline \multirow[t]{2}{*}{ Hlth } & -19.550 & -34.492 & 2.829 & 0.30 & 0.631 & 1.822 & -0.029 & 0.49 & 1.485 & 3.631 & 0.325 & 0.31 \\
\hline & $(9.691)^{* *}$ & $(3.803) * * *$ & $(0.053)^{* * *}$ & & $(0.192)^{* * *}$ & $(0.106)^{* * *}$ & $(0.001)^{* * *}$ & & $(0.985)$ & $(0.391)^{* * *}$ & $(0.006)^{* * *}$ & \\
\hline \multirow[t]{2}{*}{ Durbl } & -12.163 & -38.317 & 1.922 & 0.48 & 0.375 & 0.309 & 0.001 & 0.13 & 0.454 & 0.590 & 0.491 & 0.02 \\
\hline & $(8.591)$ & $(3.647)^{* * *}$ & $(0.048)^{* * *}$ & & $(0.138)^{* * *}$ & $(0.073)^{* * *}$ & $(0.001)^{*}$ & & $(0.507)$ & $(0.209)^{* * *}$ & $(0.003)^{* * *}$ & \\
\hline \multirow[t]{2}{*}{ Manuf } & -7.849 & -33.477 & 1.815 & 0.44 & 0.540 & 0.357 & 0.002 & 0.26 & 0.030 & 0.212 & 0.489 & 0.00 \\
\hline & $(6.581)$ & $(3.436) * * *$ & $(0.041)^{* * *}$ & & $(0.076)^{* * *}$ & $(0.051)^{* * *}$ & $(0.001)^{* * *}$ & & $(0.322)$ & $(0.196)$ & $(0.003)^{* * *}$ & \\
\hline \multirow[t]{2}{*}{ Chems } & -10.118 & -39.179 & 2.189 & 0.44 & 0.438 & 0.445 & 0.004 & 0.28 & -1.899 & -2.578 & 0.516 & 0.20 \\
\hline & $(6.737)$ & $(3.078) * * *$ & $(0.043)^{* * *}$ & & $(0.094)^{* * *}$ & $(0.057)^{* * *}$ & $(0.001)^{* * *}$ & & $(0.464)^{* * *}$ & $(0.292)^{* * *}$ & $(0.004)^{* * *}$ & \\
\hline \multirow[t]{2}{*}{ Shops } & -15.538 & -38.798 & 2.072 & 0.44 & 0.293 & 0.431 & -0.002 & 0.26 & 1.082 & 1.775 & 0.495 & 0.29 \\
\hline & $(8.436)^{*}$ & $(3.715)^{* * *}$ & $(0.048)^{* * *}$ & & $(0.089)^{* * *}$ & $(0.049)^{* * *}$ & $(0.001)^{* * *}$ & & $(0.531)^{* *}$ & $(0.212)^{* * *}$ & $(0.003)^{* * *}$ & \\
\hline \multirow[t]{2}{*}{ Mines } & -2.082 & -12.526 & 1.866 & 0.04 & 0.657 & 0.992 & -0.011 & 0.36 & -0.094 & 0.418 & 0.319 & 0.00 \\
\hline & $(5.960)$ & $(4.380) * * *$ & $(0.057)^{* * *}$ & & $(0.185)^{* * *}$ & $(0.093)^{* * *}$ & $(0.001)^{* * *}$ & & (1.097) & $(0.477)$ & $(0.006)^{* * *}$ & \\
\hline \multirow[t]{2}{*}{ Cnstr } & -8.174 & -25.494 & 1.631 & 0.25 & 0.271 & 0.137 & 0.002 & 0.05 & 1.287 & 2.292 & 0.540 & 0.16 \\
\hline & $(5.784)$ & $(3.182)^{* * *}$ & $(0.041)^{* * *}$ & & $(0.103)^{* * *}$ & $(0.072)^{* * *}$ & $(0.001)^{* * *}$ & & $(0.542)^{* *}$ & $(0.284)^{* * *}$ & $(0.004)^{* * *}$ & \\
\hline
\end{tabular}

$*$ : Significant at $10 \%$

**. Significant at $5 \%$

$* * *$ : Significant at $1 \%$ 
Appendix B

Lagged Inflation, $M / B, R O A$, and Leverage (Continues)

$\mathrm{M} / \mathrm{B}_{\mathrm{i}, \mathrm{t}}=\beta_{0}+\beta_{1} \mathrm{U}_{\mathrm{t}-1} \pi_{\mathrm{t}-1}+\beta_{2} \mathrm{E}_{\mathrm{t}-2} \pi_{\mathrm{t}-1}+\varepsilon_{\mathrm{i}, \mathrm{t}}$

$\mathrm{ROA}_{\mathrm{i}, \mathrm{t}}=\beta_{0}+\beta_{1} \mathrm{U}_{\mathrm{t}-1} \pi_{\mathrm{t}-1}+\beta_{2} \mathrm{E}_{\mathrm{t}-2} \pi_{\mathrm{t}-1}+\varepsilon_{\mathrm{i}, \mathrm{t}}$

$\mathrm{LEV}_{\mathrm{i}, \mathrm{t}}=\beta_{0}+\beta_{1} \mathrm{U}_{\mathrm{t}-1} \pi_{\mathrm{t}-1}+\beta_{2} \mathrm{E}_{\mathrm{t}-2} \pi_{\mathrm{t}-1}+\varepsilon_{\mathrm{i}, \mathrm{t}}$

where $\mathrm{U}_{\mathrm{t}-1} \pi_{\mathrm{t}-1}$ is the unexpected quarterly inflation rate at $\mathrm{t}-1, \pi_{\mathrm{t}-1}$ is the expected quarterly inflation rate at $\mathrm{t}-1, \mathrm{M} / \mathrm{B}_{\mathrm{i}, \mathrm{t}}$ is the average quarterly market to book ratio, $\mathrm{ROA}_{i, t}$ is the average quarterly return on assets, $\mathrm{LEV}_{\mathrm{i}, \mathrm{t}}$ is the average quarterly leverage ratio, and $\varepsilon_{\mathrm{i}, \mathrm{t}}$ is a residual. The residual of the cyclical- and non-cyclical industry portfolio is estimated based on the fixed-effects model in panel regression.

\begin{tabular}{|c|c|c|c|c|c|c|c|c|c|c|c|c|}
\hline \multirow{2}{*}{$\begin{array}{l}\text { Panel B } \\
\text { Industry }\end{array}$} & \multicolumn{3}{|c|}{$\mathbf{M} / \mathbf{B}$} & \multicolumn{4}{|c|}{ ROA } & \multicolumn{5}{|c|}{ LEV } \\
\hline & $\beta_{1}$ & $\boldsymbol{\beta}_{2}$ & $\boldsymbol{\beta}_{0}$ & $\mathbf{R}^{2}$ & $\beta_{1}$ & $\boldsymbol{\beta}_{2}$ & $\boldsymbol{\beta}_{0}$ & $\mathbf{R}^{2}$ & $\beta_{1}$ & $\boldsymbol{\beta}_{2}$ & $\boldsymbol{\beta}_{0}$ & $\mathbf{R}^{2}$ \\
\hline 61Q2-14Q4 & & & & & & & & & & & & \\
\hline Non-cyclical & $\begin{array}{c}-13.087 \\
(2.548)^{* * *}\end{array}$ & $\begin{array}{c}-32.644 \\
(1.898) * * *\end{array}$ & $\begin{array}{c}2.082 \\
(0.022)^{* * *}\end{array}$ & 0.13 & $\begin{array}{c}0.307 \\
(0.082)^{* * *}\end{array}$ & $\begin{array}{c}0.760 \\
(0.061)^{* * *}\end{array}$ & $\begin{array}{c}-0.006 \\
(0.001)^{* * *}\end{array}$ & 0.14 & $\begin{array}{c}0.511 \\
(0.258)^{* *}\end{array}$ & $\begin{array}{c}1.318 \\
(0.192)^{* * *}\end{array}$ & $\begin{array}{c}0.480 \\
(0.002)^{* * *}\end{array}$ & 0.01 \\
\hline Cyclical & $\begin{array}{c}-9.369 \\
(1.941)^{* * *} \\
\end{array}$ & $\begin{array}{c}-31.414 \\
(1.452)^{* * * *} \\
\end{array}$ & $\begin{array}{c}1.916 \\
(0.017)^{* * *} \\
\end{array}$ & 0.28 & $\begin{array}{c}0.427 \\
(0.042)^{* * *} \\
\end{array}$ & $\begin{array}{c}0.442 \\
(0.032)^{* * *} \\
\end{array}$ & $\begin{array}{l}-0.001 \\
(0.000) \\
\end{array}$ & 0.18 & $\begin{array}{c}0.146 \\
(0.229) \\
\end{array}$ & $\begin{array}{c}0.452 \\
(0.172)^{* * *} \\
\end{array}$ & $\begin{array}{c}0.476 \\
(0.002)^{* * *} \\
\end{array}$ & 0.00 \\
\hline 61Q2-82Q4 & & & & & & & & & & & & \\
\hline Non-cyclical & $\begin{array}{c}-22.575 \\
(5.511)^{* * *}\end{array}$ & $\begin{array}{c}-20.563 \\
(4.502)^{* * *}\end{array}$ & $\begin{array}{c}1.755 \\
(0.089)^{* * *}\end{array}$ & 0.08 & $\begin{array}{c}0.043 \\
(0.082)\end{array}$ & $\begin{array}{c}0.035 \\
(0.067)\end{array}$ & $\begin{array}{c}0.013 \\
(0.001)^{* * *}\end{array}$ & 0.00 & $\begin{array}{c}1.182 \\
(0.465)^{* * *}\end{array}$ & $\begin{array}{c}1.033 \\
(0.380)^{* * *}\end{array}$ & $\begin{array}{c}0.484 \\
(0.008)^{* * *}\end{array}$ & 0.02 \\
\hline Cyclical & $\begin{array}{c}-13.657 \\
(3.249) * * *\end{array}$ & $\begin{array}{c}-11.197 \\
(2.624)^{* * *}\end{array}$ & $\begin{array}{c}1.407 \\
(0.052)^{* * *}\end{array}$ & 0.07 & $\begin{array}{c}0.358 \\
(0.074)^{* * *}\end{array}$ & $\begin{array}{c}0.029 \\
(0.060)\end{array}$ & $\begin{array}{c}0.011 \\
(0.001)^{* * *}\end{array}$ & 0.07 & $\begin{array}{c}1.447 \\
(0.487)^{* * *}\end{array}$ & $\begin{array}{c}1.070 \\
(0.394)^{* * *}\end{array}$ & $\begin{array}{c}0.459 \\
(0.008)^{* * *}\end{array}$ & 0.01 \\
\hline 83Q1-01Q4 & & & & & & & & & & & & \\
\hline Non-cyclical & $\begin{array}{c}-10.900 \\
(3.859)^{* * *}\end{array}$ & $\begin{array}{c}-13.476 \\
(4.385)^{* * *}\end{array}$ & $\begin{array}{c}2.014 \\
(0.033)^{* * *}\end{array}$ & 0.01 & $\begin{array}{c}0.047 \\
(0.108)\end{array}$ & $\begin{array}{c}0.336 \\
(0.123)^{* * *}\end{array}$ & $\begin{array}{c}-0.004 \\
(0.001)^{* * *}\end{array}$ & 0.01 & $\begin{array}{c}1.208 \\
(0.486)^{* * *}\end{array}$ & $\begin{array}{c}1.338 \\
(0.552)^{* *}\end{array}$ & $\begin{array}{c}0.476 \\
(0.004)^{* * *}\end{array}$ & 0.00 \\
\hline Cyclical & $\begin{array}{c}-9.065 \\
(3.522)^{* * *}\end{array}$ & $\begin{array}{c}-20.431 \\
(4.002)^{* * *}\end{array}$ & $\begin{array}{c}1.883 \\
(0.030)^{* * *}\end{array}$ & 0.04 & $\begin{array}{c}0.025 \\
(0.074) \\
\end{array}$ & $\begin{array}{c}-0.219 \\
(0.084)^{* * *}\end{array}$ & $\begin{array}{c}0.002 \\
(0.001)^{* * *}\end{array}$ & 0.01 & $\begin{array}{c}1.050 \\
(0.440)^{* *}\end{array}$ & $\begin{array}{c}2.548 \\
(0.499)^{* * *}\end{array}$ & $\begin{array}{c}0.468 \\
(0.004)^{* * *}\end{array}$ & 0.01 \\
\hline 02Q1-14Q4 & & & & & & & & & & & & \\
\hline Non-cyclical & $\begin{array}{c}8.100 \\
(2.602)^{* * *}\end{array}$ & $\begin{array}{l}-4.936 \\
(3.654)\end{array}$ & $\begin{array}{c}1.993 \\
(0.024)^{* * *}\end{array}$ & 0.01 & $\begin{array}{c}0.102 \\
(0.050)^{* *}\end{array}$ & $\begin{array}{l}-0.029 \\
(0.070)\end{array}$ & $\begin{array}{c}-0.005 \\
(0.000)^{* * *}\end{array}$ & 0.00 & $\begin{array}{c}-0.659 \\
(0.311)^{* *}\end{array}$ & $\begin{array}{c}0.606 \\
(0.437)\end{array}$ & $\begin{array}{c}0.487 \\
(0.003)^{* * *}\end{array}$ & 0.00 \\
\hline Cyclical & $\begin{array}{c}10.811 \\
(2.567)^{* * *}\end{array}$ & $\begin{array}{c}-5.973 \\
(3.604)^{* * *}\end{array}$ & $\begin{array}{c}1.895 \\
(0.023)^{* * *}\end{array}$ & 0.05 & $\begin{array}{c}0.283 \\
(0.050)^{* * *}\end{array}$ & $\begin{array}{c}-0.168 \\
(0.071)^{* *}\end{array}$ & $\begin{array}{c}0.002 \\
(0.000)^{* * *}\end{array}$ & 0.08 & $\begin{array}{c}-0.820 \\
(0.199)^{* * *}\end{array}$ & $\begin{array}{r}0.399 \\
(0.280) \\
\end{array}$ & $\begin{array}{c}0.471 \\
(0.002)^{* * *}\end{array}$ & 0.01 \\
\hline
\end{tabular}

*: Significant at $10 \%$

**: Significant at 5\%

$* * *$ : Significant at $1 \%$ 
